\title{
Applications on the Apostol-Daehee numbers and polynomials associated with special numbers, polynomials, and $p$-adic integrals
}

\author{
Yilmaz Simsek ${ }^{1 *}$ (D) and Ahmet Yardimci ${ }^{1,2}$
}

${ }^{*}$ Correspondence:

ysimsek@akdeniz.edu.tr

1 Department of Mathematics,

Faculty of Science, University of

Akdeniz, Antalya, 07058, Turkey

Full list of author information is

available at the end of the article

\section{空 Springer}

\begin{abstract}
In this paper, by using $p$-adic Volkenborn integral, and generating functions, we give some properties of the Bernstein basis functions, the Apostol-Daehee numbers and polynomials, Apostol-Bernoulli polynomials, some special numbers including the Stirling numbers, the Euler numbers, the Daehee numbers, and the Changhee numbers. By using an integral equation and functional equations of the generating functions and their partial differential equations (PDEs), we give a recurrence relation for the Apostol-Daehee polynomials. We also give some identities, relations, and integral representations for these numbers and polynomials. By using these relations, we compute these numbers and polynomials. We make further remarks and observations for special polynomials and numbers, which are used to study elementary word problems in engineering and in medicine.
\end{abstract}

MSC: 12D10; 11B68; 11S40; 11S80; 26C05; 26C10; 30B40; 30C15

Keywords: Bernoulli numbers and polynomials; Apostol-Bernoulli numbers and polynomials; Daehee numbers and polynomials; Apostol-Daehee numbers; array polynomials; Stirling numbers of the first kind and the second kind; generating function; functional equation; derivative equation; Bernstein basis functions

\section{Introduction}

The special numbers and polynomials have been used in various applications in such diverse areas as mathematics, probability and statistics, mathematical physics, and engineering. For example, due to the relative freedom of some basic operations including addition, subtraction, multiplication, polynomials can be seen almost ubiquitously in engineering. They are curves that represent properties or behavior of many engineering objects or devices. For example, polynomials are used in elementary word problems to complicated problems in the sciences, approximate or curve fit experimental data, calculate beam deflection under loading, represent some properties of gases, and perform computer aided geometric design in engineering. Polynomials are used as solutions of differential equations. Polynomials represent characteristics of linear dynamic system and we also know that a ratio of two polynomials represents a transfer function of a linear dynamic system.

(c) The Author(s) 2016. This article is distributed under the terms of the Creative Commons Attribution 4.0 International License (http://creativecommons.org/licenses/by/4.0/), which permits unrestricted use, distribution, and reproduction in any medium, provided you give appropriate credit to the original author(s) and the source, provide a link to the Creative Commons license, and indicate if changes were made. 
With the help of polynomials, one defines basis functions used in finite element computation and constructs parametric curves.

In order to give some results including identities, relations, and formulas for special numbers and polynomials, we use the $p$-adic Volkenborn integral and generating function methods. We need the following formulas, relations, generating functions, and notations for families of special numbers and polynomials. Throughout this paper, we use the following notations:

Let $\mathbb{C}, \mathbb{R}, \mathbb{Z}$, and $\mathbb{N}$ be the sets of complex numbers, real numbers, integers, and positive integers, respectively, and $\mathbb{N}_{0}=\mathbb{N} \cup\{0\}$ and $\mathbb{Z}^{-}=\{-1,-2,-3, \ldots\}$. Also let $\mathbb{Z}_{p}$ be the set of $p$-adic integers. We assume that $\ln (z)$ denotes the principal branch of the multi-valued function $\ln (z)$ with the imaginary part $\operatorname{Im}(\ln (z))$ constrained by $-\pi<\operatorname{Im}(\ln (z)) \leq \pi$. Furthermore, $0^{n}=1$ if $n=0$, and $0^{n}=0$ if $n \in \mathbb{N}$. We have

$$
\left(\begin{array}{l}
x \\
v
\end{array}\right)=\frac{x(x-1) \cdots(x-v+1)}{v !}=\frac{(x)_{v}}{v !} \quad\left(x \in \mathbb{C} ; v \in \mathbb{N}_{0}\right)
$$

(cf. [1-47], and the references cited therein).

There are many methods and techniques for investigating and constructing generating functions for special polynomials and numbers. One of the most important techniques is the $p$-adic Volkenborn integral on $\mathbb{Z}_{p}$. In [19], Kim constructed the $p$-adic $q$-Volkenborn integration. By using this integral, we derive some identities, and relations for the special polynomials. We now briefly give some definitions and properties of this integral.

Let $f \in U D\left(\mathbb{Z}_{p}\right)$, the set of uniformly differentiable functions on $\mathbb{Z}_{p}$. The $p$-adic $q$ Volkenborn integration of $f$ on $\mathbb{Z}_{p}$ is defined by Kim [19] as follows:

$$
\int_{\mathbb{Z}_{p}} f(x) d \mu_{q}(x)=\lim _{N \rightarrow \infty} \frac{1}{\left[p^{N}\right]_{q}} \sum_{x=0}^{p^{N}-1} f(x) q^{x},
$$

where

$$
[x]= \begin{cases}\frac{1-q^{x}}{1-q}, & q \neq 1 \\ x, & q=1\end{cases}
$$

and $\mu_{q}(x)$ denotes the $q$-distribution on $\mathbb{Z}_{p}$, which is given by

$$
\mu_{q}\left(x+p^{N} \mathbb{Z}_{p}\right)=\frac{q^{x}}{\left[p^{N}\right]_{q}},
$$

where $q \in \mathbb{C}_{p}$ with $|1-q|_{p}<1$ (cf. [19]).

If $q \rightarrow 1$ in (1.1), then we have the bosonic $p$-adic integral ( $p$-adic Volkenborn integral), which is given by $(c f .[19,45])$

$$
\int_{\mathbb{Z}_{p}} f(x) d \mu_{1}(x)=\lim _{N \rightarrow \infty} \frac{1}{p^{N}} \sum_{x=0}^{p^{N}-1} f(x),
$$

where

$$
\mu_{1}\left(x+p^{N} \mathbb{Z}_{p}\right)=\frac{1}{p^{N}} .
$$


By using this integral, the Bernoulli polynomials are given by

$$
B_{n}(x)=\int_{\mathbb{Z}_{p}}(x+y)^{n} d \mu_{1}(y)
$$

(cf. [19, 20, 37, 45, 47], and the references cited therein).

The Bernoulli polynomials $B_{n}(x)$ are also defined by means of the following generating function:

$$
F_{B}(t, x)=\frac{t}{e^{t}-1} e^{t x}=\sum_{n=0}^{\infty} B_{n}(x) \frac{t^{n}}{n !}
$$

with $B_{n}(0)=B_{n}$, which denotes the Bernoulli numbers (of the first kind) $(c f .[9,20,27-30$, $35,37,46,47]$, and the references cited therein).

If $q \rightarrow-1$ in (1.1), then we have the fermionic $p$-adic integral on $\mathbb{Z}_{p}$ given by ( $c f$. [20]):

$$
\int_{\mathbb{Z}_{p}} f(x) d \mu_{-1}(x)=\lim _{N \rightarrow \infty} \sum_{x=0}^{p^{N}-1}(-1)^{x} f(x),
$$

where $p \neq 2$ and

$$
\mu_{-1}\left(x+p^{N} \mathbb{Z}_{p}\right)=(-1)^{x}
$$

(cf. [20]). By using (1.4), we have the Witt formula for the Euler numbers $E_{n}$ as follows:

$$
E_{n}(x)=\int_{\mathbb{Z}_{p}}(x+y)^{n} d \mu_{-1}(y)
$$

(cf. [9, 20, 37, 47], and the references cited therein).

The Euler polynomials $E_{n}(x)$ are also defined by means of the following generating function:

$$
F_{E}(t, x)=\frac{2}{e^{t}+1} e^{t x}=\sum_{n=0}^{\infty} E_{n}(x) \frac{t^{n}}{n !}
$$

with $E_{n}(0)=E_{n}$, which denotes the Euler numbers (of the first kind) $(c f .[9,20,27-30,35$, $37,46,47]$, and the references cited therein).

The $\lambda$-Bernoulli numbers and polynomials have been studied in different sets. For instance on the set of complex numbers, we assume that $\lambda \in \mathbb{C}$ and on set of $p$-adic numbers or $p$-adic integrals, we assume that $\lambda \in \mathbb{Z}_{p}$.

The Apostol-Bernoulli polynomials $B_{n}(x ; \lambda)$ are defined by means of the following generating function:

$$
F_{A}(t, x ; \lambda)=\frac{t}{\lambda e^{t}-1} e^{t x}=\sum_{n=0}^{\infty} B_{n}(x ; \lambda) \frac{t^{n}}{n !} .
$$

For $x=0$, we have the Apostol-Bernoulli numbers

$$
B_{n}(\lambda)=B_{n}(0 ; \lambda)
$$


and

$$
B_{n}=B_{n}(0 ; 1)
$$

which denotes the Bernoulli numbers (of the first kind) $(c f .[9,20,27-30,35,37,46,47]$, and the references cited therein).

The $\lambda$-Stirling numbers of the second kind are defined by means of the following generating function:

$$
F_{L S}(t, v ; \lambda)=\frac{\left(\lambda e^{t}-1\right)^{v}}{v !}=\sum_{n=0}^{\infty} S_{2}(n, v ; \lambda) \frac{t^{n}}{n !}
$$

(cf. [27]; see also [28, 35, 38, 39, 46], and the references cited therein).

In [4], the Stirling number of the second kind $S_{2}(n, k)$ are defined in combinatorics: the Stirling numbers of the second kind are the number of ways to partition a set of $n$ objects into $k$ groups. These numbers are defined by means of the following generating function:

$$
F_{S}(t, v)=\frac{\left(e^{t}-1\right)^{v}}{v !}=\sum_{n=0}^{\infty} S_{2}(n, v) \frac{t^{n}}{n !}
$$

( $c f$. [1-47], and the references cited therein). By using the above generating function, these numbers are computed by the following explicit formula:

$$
S_{2}(n, v)=\frac{1}{v !} \sum_{j=0}^{v}\left(\begin{array}{l}
v \\
j
\end{array}\right)(-1)^{j}(v-j)^{n}
$$

Setting $\lambda=1$ in (1.6), we have

$$
S_{2}(n, v ; 1)=S_{2}(n, v)
$$

(cf. [1-47], and the references cited therein).

The Stirling numbers of the first kind $s_{1}(n, v)$ are defined by means of the following generating function:

$$
F_{s 1}(t, k)=\frac{(\log (1+t))^{k}}{k !}=\sum_{n=0}^{\infty} s_{1}(n, k) \frac{t^{n}}{n !}
$$

(cf. $[3,4,13,19,34,39]$, and the references cited therein).

The Bernstein basis functions $B_{k}^{n}(x)$ are defined as follows:

$$
B_{k}^{n}(x)=\left(\begin{array}{l}
n \\
k
\end{array}\right) x^{k}(1-x)^{n-k} \quad\left(x \in[0,1] ; n, k \in \mathbb{N}_{0}\right),
$$

where $k=0,1, \ldots, n$ and

$$
\left(\begin{array}{l}
n \\
k
\end{array}\right)=\frac{n !}{k !(n-k) !}
$$

(cf. $[1,8,21,26,40])$, and the references cited therein. 
The Bernstein basis functions can also be defined by means of the following generating functions:

$$
f_{\mathbb{B}, k}(x, t)=\frac{t^{k} x^{k} e^{(1-x) t}}{k !}=\sum_{n=0}^{\infty} B_{k}^{n}(x) \frac{t^{n}}{n !}
$$

where $k=0,1, \ldots, n$ and $t \in \mathbb{C}$ and $x \in[0,1](c f .[1,21,40,44])$ and see also the references cited in each of these earlier works.

The Bernoulli polynomials of the second kind $b_{n}(x)$ are defined by means of the following generating function:

$$
F_{b 2}(t, x)=\frac{t}{\log (1+t)}(1+t)^{x}=\sum_{n=0}^{\infty} b_{n}(x) \frac{t^{n}}{n !}
$$

(cf. [34], pp.113-117, and the references cited therein).

The Bernoulli numbers of the second kind $b_{n}(0)$ are defined by means of the following generating function:

$$
F_{b 2}(t)=\frac{t}{\log (1+t)}=\sum_{n=0}^{\infty} b_{n}(0) \frac{t^{n}}{n !} .
$$

The numbers $b_{n}(0)$ are known as the Cauchy numbers $[12,34]$.

The Daehee polynomials are defined by means of the following generating function:

$$
F_{D}(t, x)=\frac{\log (1+t)}{t}(1+t)^{x}=\sum_{n=0}^{\infty} D_{n}(x) \frac{t^{n}}{n !},
$$

with

$$
D_{n}=D_{n}(0)
$$

denotes the so-called Daehee numbers $(c f .[6,10,13,15,16,24,31,32]$, and the references cited therein).

The Changhee polynomials are defined by means of the following generating function:

$$
F_{C}(t, x)=\frac{2(1+t)^{x}}{2+t} \sum_{n=0}^{\infty} C h_{n}(x) \frac{t^{n}}{n !},
$$

with

$$
C h_{n}=C h_{n}(0)
$$

denotes the so-called Changhee numbers ( $c f .[16,25,47]$, and the references cited therein).

\section{Theorem 1}

$$
\int_{\mathbb{Z}_{p}}\left(\begin{array}{l}
x \\
j
\end{array}\right) d \mu_{1}(x)=\frac{(-1)^{j}}{j+1} .
$$


Proof of Theorem 1 was given by Schikhof [45].

\section{Theorem 2}

$$
\int_{\mathbb{Z}_{p}}\left(\begin{array}{l}
x \\
j
\end{array}\right) d \mu_{-1}(x)=\frac{(-1)^{j}}{2^{j}} .
$$

Proof of Theorem 2 was given by Kim et al. [13] and [16].

Applying the bosonic $p$-adic integral, the Witt formula for the Daehee numbers and polynomials are given by Kim et al. [16] as follows, respectively:

$$
D_{n}=\int_{\mathbb{Z}_{p}}(x)_{n} d \mu_{1}(x)
$$

and

$$
D_{n}(y)=\int_{\mathbb{Z}_{p}}(y+x)_{n} d \mu_{1}(x)
$$

Applying the fermionic $p$-adic integral, the Witt formula for the Changhee numbers and polynomials are given by Kim et al. [16] as follows, respectively:

$$
C h_{n}=\int_{\mathbb{Z}_{p}}(x)_{n} d \mu_{-1}(x)
$$

and

$$
C h_{n}(y)=\int_{\mathbb{Z}_{p}}(y+x)_{n} d \mu_{-1}(x)
$$

Remark 1 Many applications of the fermionic and bosonic $p$-adic integral on $\mathbb{Z}_{p}$ have been given by T Kim and DS Kim first, Jang, Rim, Dolgy, Kwon, Seo, Lim and the others gave various novel identities, relations and formulas in some special numbers and polynomials (cf. [6-25, 35, 37, 38, 42, 45, 47], and the references cited therein).

The $\lambda$-Bernoulli polynomials $\mathfrak{B}_{n}(x ; \lambda)$ are defined by means of the following generating function:

$$
F_{\mathfrak{B}}(t, x ; \lambda, k)=\left(\frac{\log \lambda+t}{\lambda e^{t}-1}\right)^{k} e^{t x}=\sum_{n=0}^{\infty} \mathfrak{B}_{n}^{(k)}(x ; \lambda) \frac{t^{n}}{n !}
$$

(cf. [22]).

In [43] and [42], Simsek, by using the $p$-adic Volkenborn integral on $\mathbb{Z}_{p}$, defined the $\lambda$ Apostol-Daehee numbers and polynomials, $\mathfrak{D}_{n}(x ; \lambda)$ by means of the following generating functions:

$$
G(t, x ; \lambda)=\frac{\log \lambda+\log (1+\lambda t)}{\lambda(1+\lambda t)-1}(1+\lambda t)^{x}=\sum_{n=0}^{\infty} \mathfrak{D}_{n}(x ; \lambda) \frac{t^{n}}{n !} .
$$


Observe that substituting $\lambda=1$ into $(1.19), \mathfrak{D}_{n}(x ; 1)=D_{n}(x)$, the Daehee Polynomials $(c f$. $[5,17])$. By using (1.19), we also have the following formula:

$$
D_{n}(x ; \lambda)=\sum_{k=0}^{n}\left(\begin{array}{l}
n \\
k
\end{array}\right)(x)_{n-k} D_{k}(\lambda)
$$

(cf. [42]; see also [43]).

A relation between the $\lambda$-Bernoulli polynomials $\mathfrak{B}_{n}(x ; \lambda)$, the Apostol-Daehee polynomials $\mathfrak{D}_{n}(x ; \lambda)$ and the Stirling numbers of the second kind is given by the following theorem.

\section{Theorem 3}

$$
\mathfrak{B}_{m}(x ; \lambda)=\sum_{n=0}^{m} \frac{1}{\lambda^{n}} S_{2}(m, n) \mathfrak{D}_{n}(x ; \lambda) .
$$

The proof of (1.20) was given by the first author in [43].

Observe that $G\left(\frac{e^{z}-1}{\lambda} ; \lambda\right)$ is a generating function for the $\lambda$-Bernoulli numbers $(c f$. [22]). We also observe that $G\left(e^{z}-1 ; 1\right)$ is a generating function for the Bernoulli numbers.

Substituting $\lambda=1$ into (1.19), we obtain

$$
G(t ; 1)=\frac{\log (1+t)}{t} .
$$

Observe that $G(t ; 1)$ is a generating function for the Daehee numbers $(c f .[16,17])$.

We summarize our results as follows.

In Section 2, we give some identities, relations, and formulas including the ApostolDaehee numbers and polynomials of higher order, the Changhee numbers and polynomials and the Stirling numbers, the $\lambda$-Bernoulli polynomials, the $\lambda$-Apostol-Daehee polynomials and the Bernstein basis functions.

In Section 3, we give an integral representation for the Apostol-Daehee polynomials.

In Section 4, we introduce further remarks and observations on these numbers, polynomials, and their applications.

\section{Identities}

By using the above generating functions, we get some identities and relation. In [43] and [42], Simsek gave derivative formulas for the $\lambda$-Apostol-Daehee polynomials, $\mathfrak{D}_{n}(x ; \lambda)$. Here we give another derivative formula for these polynomials. We also give a relation between the $\lambda$-Bernoulli polynomials, the $\lambda$-Apostol-Daehee polynomials and the Bernstein polynomials.

Theorem 4 Let $n \in \mathbb{N}_{0}$, then we have

$$
\frac{\partial}{\partial x} \mathfrak{D}_{n+1}(x ; \lambda)=\sum_{j=0}^{n}(-1)^{j} j !\left(\begin{array}{c}
n+1 \\
j+1
\end{array}\right) \lambda^{j+1} \mathfrak{D}_{n-j}(x ; \lambda) .
$$

Proof We can take the derivative of equation (1.19) with respect to $x$, we obtain the following partial differential equation:

$$
\frac{\partial}{\partial x} G(t, x ; \lambda)=G(t, x ; \lambda) \log (1+\lambda t) .
$$


By using the above partial differential equation with (1.19), we get

$$
\sum_{n=0}^{\infty} \frac{\partial}{\partial x} \mathfrak{D}_{n}(x ; \lambda) \frac{t^{n}}{n !}=\sum_{n=0}^{\infty}(-1)^{n} \lambda^{n+1} \frac{t^{n+1}}{n+1} \sum_{n=0}^{\infty} \mathfrak{D}_{n}(x ; \lambda) \frac{t^{n}}{n !}
$$

Therefore

$$
\sum_{n=0}^{\infty} \frac{\partial}{\partial x} \mathfrak{D}_{n+1}(x ; \lambda) \frac{t^{n}}{(n+1) !}=\sum_{n=0}^{\infty}\left(\sum_{j=0}^{n} \frac{(-1)^{j} \lambda^{j+1}}{j+1} \frac{\mathfrak{D}_{n-j}(x ; \lambda)}{(n-j) !}\right) t^{n} .
$$

Comparing the coefficients of $t^{n}$ on both sides of the above equation, we arrive at the desired result.

Theorem 5 Let $n \in \mathbb{N}_{0}$. Starting with

$$
\mathfrak{D}_{0}(x ; \lambda)=\frac{\log \lambda}{\lambda-1}
$$

we have

$$
\frac{(\lambda-1)}{n+1} \mathfrak{D}_{n+1}(x ; \lambda)+\lambda^{2} \mathfrak{D}_{n}(x ; \lambda)=\frac{\log \lambda}{n+1} \lambda^{n+1}(x)_{n+1}+\lambda^{n+1} n ! \sum_{k=0}^{n} \frac{(-1)^{k}(x)_{n-k}}{(k+1)(n-k) !} .
$$

Proof By using (1.19) with the definition of the logarithmic function, we get

$$
\begin{aligned}
& (\lambda-1) \sum_{n=0}^{\infty} \frac{1}{n+1} \mathfrak{D}_{n+1}(x ; \lambda) \frac{t^{n}}{n !}+\lambda^{2} \sum_{n=0}^{\infty} \mathfrak{D}_{n}(x ; \lambda) \frac{t^{n}}{n !} \\
& =\log \lambda \sum_{n=0}^{\infty} \frac{(x)_{n}}{n+1} \frac{\lambda^{n+1} t^{n}}{n !}+\sum_{n=0}^{\infty}(-1)^{n} \frac{\lambda^{n+1} t^{n}}{n+1} \sum_{n=0}^{\infty}(x)_{n} \frac{\lambda^{n} t^{n}}{n !}
\end{aligned}
$$

By using the Cauchy product in the right side of the above equation, we get

$$
\begin{aligned}
& (\lambda-1) \sum_{n=0}^{\infty} \frac{1}{n+1} \mathfrak{D}_{n+1}(x ; \lambda) \frac{t^{n}}{n !}+\lambda^{2} \sum_{n=0}^{\infty} \mathfrak{D}_{n}(x ; \lambda) \frac{t^{n}}{n !} \\
& \quad=\log \lambda \sum_{n=0}^{\infty} \frac{(x)_{n}}{n+1} \frac{\lambda^{n+1} t^{n}}{n !}+\sum_{n=0}^{\infty}\left(n ! \sum_{k=0}^{n} \frac{(-1)^{k} \lambda^{n+1}(x)_{n-k}}{(k+1)(n-k) !}\right) \frac{t^{n}}{n !}
\end{aligned}
$$

After some elementary calculations and comparing the coefficients of $\frac{t^{n}}{n !}$ on both sides of the above equation, we arrive at the desired result.

Setting $n=1$ into (2.1), we compute a few values of the polynomials $\mathfrak{D}_{n}(x ; \lambda)$ as follows:

$$
\mathfrak{D}_{1}(x ; \lambda)=\frac{\lambda \log \lambda}{\lambda-1} x+\frac{(1-\log \lambda) \lambda^{2}-\lambda}{(\lambda-1)^{2}} .
$$

\section{Theorem 6}

$$
(1-y)^{m} \mathfrak{B}_{m}\left(x+\frac{y}{1-y} ; \lambda\right)=\sum_{j=0}^{m} B_{j}^{m}(y) \sum_{n=0}^{m-j} \frac{1}{\lambda^{n}} \mathfrak{D}_{n}(x ; \lambda) S_{2}(m-j, n) .
$$


Proof Substituting

$$
t=\frac{e^{z(1-y)}-1}{\lambda}
$$

into (1.19) and combining with (1.18), we get the following functional equation:

$$
F_{\mathfrak{B}}\left((1-y) z, x+\frac{y}{1-y} ; \lambda, 1\right)=\sum_{m=0}^{\infty} y^{m} \frac{z^{m}}{m !} \sum_{n=0}^{\infty} \frac{1}{\lambda^{n}} \mathfrak{D}_{n}(x ; \lambda) \frac{\left(e^{z(1-y)}-1\right)^{n}}{n !}
$$

Combining the above equation with (1.7), we get

$$
\begin{aligned}
& \sum_{m=0}^{\infty}(1-y)^{m} \mathfrak{B}_{m}^{(1)}\left(x+\frac{y}{1-y} ; \lambda\right) \frac{z^{m}}{m !} \\
& \quad=\sum_{m=0}^{\infty} y^{m} \frac{z^{m}}{m !} \sum_{n=0}^{\infty} \frac{1}{\lambda^{n}} \mathfrak{D}_{n}(x ; \lambda) \sum_{m=0}^{\infty} S_{2}(m, n)(1-y)^{m} \frac{z^{m}}{m !}
\end{aligned}
$$

Since $n>m, S_{2}(m, n)=0$, we have

$$
\begin{aligned}
& \sum_{m=0}^{\infty}(1-y)^{m} \mathfrak{B}_{m}^{(1)}\left(x+\frac{y}{1-y} ; \lambda\right) \frac{z^{m}}{m !} \\
& \quad=\sum_{m=0}^{\infty} y^{m} \frac{z^{m}}{m !} \sum_{m=0}^{\infty} \sum_{n=0}^{m} \frac{1}{\lambda^{n}} \mathfrak{D}_{n}(x ; \lambda) S_{2}(m, n)(1-y)^{m} \frac{z^{m}}{m !}
\end{aligned}
$$

Therefore

$$
\begin{aligned}
& \sum_{m=0}^{\infty}(1-y)^{m} \mathfrak{B}_{m}^{(1)}\left(x+\frac{y}{1-y} ; \lambda\right) \frac{z^{m}}{m !} \\
& \quad=\sum_{m=0}^{\infty} \sum_{j=0}^{m} B_{j}^{m}(y) \sum_{n=0}^{m-j} \frac{1}{\lambda^{n}} \mathfrak{D}_{n}(x ; \lambda) S_{2}(m-j, n) \frac{z^{m}}{m !} .
\end{aligned}
$$

Comparing the coefficients of $\frac{z^{m}}{m !}$ on both sides of the above equation, we arrive at the desired result.

Combining (1.20) with (2.2), we get the following theorem.

\section{Theorem 7}

$$
(1-y)^{m} \mathfrak{B}_{m}\left(x+\frac{y}{1-y} ; \lambda\right)=\sum_{j=0}^{m} B_{j}^{m}(y) \mathfrak{B}_{m-j}(x ; \lambda)
$$

\section{Theorem 8}

$$
\int_{0}^{1}(1-y)^{m} \mathfrak{B}_{m}\left(x+\frac{y}{1-y} ; \lambda\right) d y=\frac{1}{m+1} \sum_{j=0}^{m} \mathfrak{B}_{m-j}(x ; \lambda) .
$$


Proof Integrating both sides of equation (2.3) from 0 to 1 with respect to $y$, we obtain

$$
\int_{0}^{1}(1-y)^{m} \mathfrak{B}_{m}^{(k)}\left(x+\frac{y}{1-y} ; \lambda\right) d y=\sum_{j=0}^{m} \mathfrak{B}_{m-j}(x ; \lambda) \int_{0}^{1} B_{j}^{m}(y) d y .
$$

Since

$$
\int_{0}^{1} B_{j}^{m}(y) d y=\frac{1}{m+1}
$$

(cf. [26]), we get

$$
\int_{0}^{1}(1-y)^{m} \mathfrak{B}_{m}^{(k)}\left(x+\frac{y}{1-y} ; \lambda\right) d y=\frac{1}{m+1} \sum_{j=0}^{m} \mathfrak{B}_{m-j}(x ; \lambda) .
$$

Therefore, the proof of the theorem is completed.

By using (1.19), we derive the following functional equation:

$$
G(t, x+y ; \lambda)=(1+\lambda t)^{y} G(t, x ; \lambda) .
$$

By using the above functional equations, we have the following theorem.

\section{Theorem 9}

$$
\mathfrak{D}_{n}(x+y ; \lambda)=\sum_{j=0}^{n}\left(\begin{array}{l}
n \\
j
\end{array}\right)(y)_{j} \mathfrak{D}_{n-j}(x ; \lambda) \lambda^{j}
$$

\section{Integral representation for the Apostol-Daehee polynomials}

In [43], Simsek defined the Apostol-Daehee polynomials $\mathfrak{D}_{n}^{(k)}(x ; \lambda)$ of higher order $k$ by means of the following generating function:

$$
F_{\mathfrak{D}}(t, x ; \lambda, k)=\left(\frac{\log \lambda+\log (1+\lambda t)}{\lambda^{2} t+\lambda-1}\right)^{k}(1+\lambda t)^{x}=\sum_{n=0}^{\infty} \mathfrak{D}_{n}^{(k)}(x ; \lambda) \frac{t^{n}}{n !} .
$$

Setting $x=0$ in (3.1) gives the Apostol-Daehee numbers $\mathfrak{D}_{n}^{(k)}(\lambda)$ of higher order $k$ :

$$
\mathfrak{D}_{n}^{(k)}(\lambda)=\mathfrak{D}_{n}^{(k)}(0 ; \lambda)
$$

By the same method as in [18], we give a multiple bosonic $p$-adic integral for the ApostolDaehee polynomials of higher order $\mathfrak{D}_{n}^{(k)}(x ; \lambda)$ in the following form:

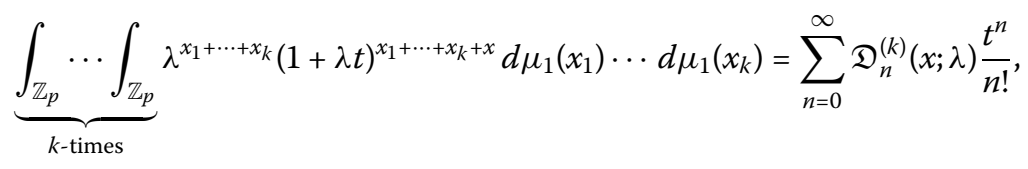


where $\lambda \in \mathbb{Z}_{p}$. Setting $\lambda=1$ in (3.2), we have

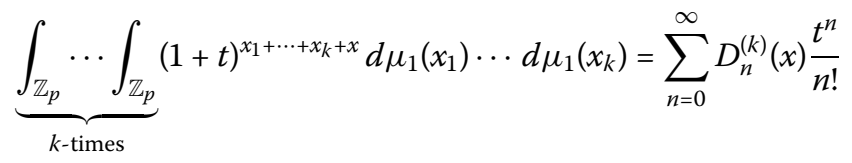

(cf. [18]; see also [16, 18], and the references therein).

Substituting $k=1$ into (3.2), we have

$$
\int_{\mathbb{Z}_{p}} \lambda^{x_{1}}(1+\lambda t)^{x+x_{1}} d \mu_{1}\left(x_{1}\right)=\sum_{n=0}^{\infty} \mathfrak{D}_{n}(x ; \lambda) \frac{t^{n}}{n !}
$$

where $\lambda \in \mathbb{Z}_{p}(c f$. [43]).

By applying the bosonic $p$-adic integral with (1.16) to the polynomials $\mathfrak{D}_{n}(x+y ; \lambda)$, we get

$$
\int_{\mathbb{Z}_{p}} \mathfrak{D}_{n}(x+y ; \lambda) d \mu_{1}(y)=\sum_{j=0}^{n}\left(\begin{array}{l}
n \\
j
\end{array}\right) D_{j} \mathfrak{D}_{n-j}(x ; \lambda) \lambda^{j},
$$

where $\lambda \in \mathbb{Z}_{p}$.

In [16], p.5972, equation (2.10), [18], Kim et al. gave an explicit form for the Daehee numbers as follows:

$$
D_{n}=\sum_{l=0}^{n} s_{1}(n, l) B_{l}
$$

Substituting this identity into (3.3), we get the following theorem.

Theorem 10 Let $\lambda \in \mathbb{Z}_{p}$. We have

$$
\int_{\mathbb{Z}_{p}} \mathfrak{D}_{n}(x+y ; \lambda) d \mu_{1}(y)=\sum_{j=0}^{n} \sum_{l=0}^{j}\left(\begin{array}{l}
n \\
j
\end{array}\right) \lambda^{j} s_{1}(j, l) B_{l} \mathfrak{D}_{n-j}(x ; \lambda) .
$$

By applying the fermionic $p$-adic Volkenborn integral with (1.17) to the polynomials $\mathfrak{D}_{n}(x+y ; \lambda)$, we get

$$
\int_{\mathbb{Z}_{p}} \mathfrak{D}_{n}(x+y ; \lambda) d \mu_{-1}(y)=\sum_{j=0}^{n}\left(\begin{array}{l}
n \\
j
\end{array}\right) C h_{j} \mathfrak{D}_{n-j}(x ; \lambda) \lambda^{j} .
$$

In [16], p.5972, equation (2.10), [18], Kim et al. gave explicit form for the Changhee numbers as follows:

$$
C h_{n}=\sum_{l=0}^{n} s_{1}(n, l) E_{l}
$$

Substituting the above formula into (3.4), we get the following theorem. 
Theorem 11 Let $\lambda \in \mathbb{Z}_{p}$. We have

$$
\int_{\mathbb{Z}_{p}} \mathfrak{D}_{n}(x+y ; \lambda) d \mu_{-1}(y)=\sum_{j=0}^{n} \sum_{l=0}^{j}\left(\begin{array}{l}
n \\
j
\end{array}\right) \lambda^{j} s_{1}(j, l) E_{l} \mathfrak{D}_{n-j}(x ; \lambda) .
$$

\section{Further remark and observations on special polynomials}

Polynomials appear in many branches of mathematics and science. For instance, polynomials are used to form polynomial equations, which encode a wide range of problems, from elementary world problems to complicated problems in the sciences, in settings ranging from basic chemistry and physics to economics and social science, in calculus and numerical analysis to approximate other functions $(c f$. $[33,48])$. Therefore, many authors have studied and investigated special polynomials and special numbers. There are various applications of these polynomials and numbers in many branches of not only in mathematics and mathematical physics, but also in computer and in engineering science with real world problems including the combinatorial sums, combinatorial numbers such as the Bernoulli numbers and polynomials, the Euler numbers and polynomials, the Stirling numbers, the Changhee numbers and polynomials, the Daehee numbers and polynomials and the others. Especially, the Bernstein polynomials are also used in many branches of mathematics, particularly including statistics, probability, combinatorics, computer algorithm, discrete mathematics and CAGD. The Bernstein basis functions are applied in real world problems to construct the theory of the Bezier curves $(c f$. $[33,48]$, and the references therein).

Such special polynomials and numbers including the above-mentioned ones have found diverse applications in many research fields other than mathematics such as mathematical physics, computer science, and engineering. That is, in engineering, polynomials are used to model real phenomena. For instance, aerospace engineers use polynomials to model the projections of jet rockets. Scientists use polynomials in many formulas including gravity, temperature, and distance equations. In social science, economists need an understanding of polynomials to forecast future market patterns ( $c f$. [33, 48], and the references therein). Polynomials are also used in analysis of ambulatory blood pressure measurements and also biostatistics problems (cf. [49]).

It is well known that there are many application of the $p$-adic integral on $\mathbb{Z}_{p}$, one of the best known applications is to construct generating functions for special numbers and polynomials. The other applications are in $p$-adic analysis, in $q$-analysis, in quantum groups, in spectra of the $q$-deformed oscillator and in science ( $c f$. $[11,45,50])$.

How can one give applications in investigating engineering and medicine related problems by using the Apostol-Daehee numbers and polynomials, and the Changhee numbers and polynomials with the $p$-integral on $\mathbb{Z}_{p}$ ?

Competing interests

The authors declare that they have no competing interests.

Authors' contributions

All authors contributed equally to the writing of this paper. All authors read and approved the final manuscript.

Author details

${ }^{1}$ Department of Mathematics, Faculty of Science, University of Akdeniz, Antalya, 07058, Turkey. ${ }^{2}$ Department of Biostatistics and Medical Informatics, Faculty of Medicine, Akdeniz University, Antalya, Turkey. 


\section{Acknowledgements}

The paper was supported by the Scientific Research Project Administration of Akdeniz University. The authors would like to thank the reviewers for their comments, which improved the previous version of this paper.

Received: 29 September 2016 Accepted: 22 November 2016 Published online: 29 November 2016

\section{References}

1. Acikgoz, M, Araci, S: On generating function of the Bernstein polynomials. AlP Conf. Proc. 1281, 1141-1143 (2010)

2. Charalambides, CA: Enumerative Combinatorics. Chapman \& Hall/CRC, Boca Raton (2002)

3. Cigler, J: Fibonacci polynomials and central factorial numbers. Preprint

4. Comtet, L: Advanced Combinatorics: The Art of Finite and Infinite Expansions. Reidel, Dordrecht (1974) (translated from the French by JW Nienhuys)

5. El-Desouky, BS, Mustafa, A: New results on higher-order Daehee and Bernoulli numbers and polynomials. arXiv:1503.00104v1

6. Do, Y, Lim, D: On (h, q)-Daehee numbers and polynomials. Adv. Differ. Equ. 2015, 107 (2015)

7. Dolgy, DV, Kim, T, Kwon, HI, Seo, J-J: A note on degenerate Bell numbers and polynomials associated with $p$-adic integral on $\mathbb{Z}_{p}$. Adv. Stud. Contemp. Math. 26(3), 457-466 (2016)

8. Goldman, RN: Identities for the univariate and bivariate Bernstein basis functions. In: Paeth, A (ed.) Graphics Gems V, pp. 149-162. Academic Press, San Diego (1995)

9. Jang, LC, Kim, T: A new approach to q-Euler numbers and polynomials. J. Concr. Appl. Math. 6, 159-168 (2008)

10. Jang, LC, Pak, HK: Non-Archimedean integration associated with q-Bernoulli numbers. Proc. Jangjeon Math. Soc. 5(2), 125-129 (2002)

11. Khrennikov, A: p-Adic Valued Distributions in Mathematical Physics. Kluwer Academic, Dordrecht (1994)

12. Kim, D-S, Kim, T, Seo, J-J, Komatsu, T: Barnes' multiple Frobenius-Euler and poly-Bernoulli mixed-type polynomials. Adv. Differ. Equ. 2014, 92 (2014)

13. Kim, DS, Kim, T: Daehee numbers and polynomials. Appl. Math. Sci. (Ruse) 7(120), 5969-5976 (2013)

14. Kim, DS, Kim, T: Some new identities of Frobenius-Euler numbers and polynomials. J. Inequal. Appl. 2012,307 (2012)

15. Kim, DS, Kim, T, Lee, S-H, Seo, J-J: A note on the lambda-Daehee polynomials. Int. J. Math. Anal. 7(62), 3069-3080 (2013)

16. Kim, DS, Kim, T, Seo, J-J: A note on Changhee numbers and polynomials. Adv. Stud. Theor. Phys. 7, $993-1003$ (2013)

17. Kim, DS, Kim, T, Lee, SH, Seo, J-J: Higher-order Daehee numbers and polynomials. Int. J. Math. Anal. 8(6), 273-283 (2014)

18. Kim, DS, Kim, T, Kwon, HI: Identities of some special mixed-type polynomials. arXiv:1406.2124v1

19. Kim, T: q-Volkenborn integration. Russ. J. Math. Phys. 19, 288-299 (2002)

20. Kim, T: $q$-Euler numbers and polynomials associated with $p$-adic $q$-integral and basic $q$-zeta function. Trends Math. Inf. Cent. Math. Sci. 9, 7-12 (2006)

21. Kim, T: Some identities on the $q$-integral representation of the product of several $q$-Bernstein-type polynomials. Abstr. Appl. Anal. 2011, Article ID 634675 (2011)

22. Kim, T, Rim, S-H, Simsek, Y, Kim, D: On the analogs of Bernoulli and Euler numbers, related identities and zeta and L-functions. J. Korean Math. Soc. 45(2), 435-453 (2008)

23. Kim, T, Kim, DS, Kwon, HI, Dolgy, DV, Seo, J-J: Degenerate falling factorial polynomials. Adv. Stud. Contemp. Math. 26(3), 481-499 (2016)

24. Lim, D: On the twisted modified q-Daehee numbers and polynomials. Adv. Stud. Theor. Phys. 9(4), $199-211$ (2015)

25. Lim, D, Qi, F: On the Appell type $\lambda$-Changhee polynomials. J. Nonlinear Sci. Appl. 9, 1872-1876 (2016)

26. Lorentz, GG: Bernstein Polynomials. Chelsea, New York (1986)

27. Luo, QM, Srivastava, HM: Some generalizations of the Apostol-Genocchi polynomials and the Stirling numbers of the second kind. Appl. Math. Comput. 217, 5702-5728 (2011)

28. Ozden, H, Simsek, Y: Modification and unification of the Apostol-type numbers and polynomials and their applications. Appl. Math. Comput. 235, 338-351 (2014)

29. Ozden, H, Cangul, IN, Simsek, Y: Remarks on q-Bernoulli numbers associated with Daehee numbers. Adv. Stud. Contemp. Math. 18(1), 41-48 (2009)

30. Ozden, H, Simsek, Y, Srivastava, HM: A unified presentation of the generating functions of the generalized Bernoulli, Euler and Genocchi polynomials. Comput. Math. Appl. 60, 2779-2787 (2010)

31. Park, J-W: On a $q$-analogue of $(h, q)$-Daehee numbers and polynomials of higher order. J. Comput. Anal. Appl. 21(1), 769-777 (2016)

32. Park, J-W, Rim, S-H, Kwon, J: The hyper-geometric Daehee numbers and polynomials. Turk. J. Anal. Number Theory 1(1), 59-62 (2013)

33. Reinard, JC: Introduction to Communication Research. McGraw-Hill, New York (2001)

34. Roman, S: The Umbral Calculus. Dover, New York (2005)

35. Simsek, Y: Special numbers on analytic functions. Appl. Math. (Irvine) 5, 1091-1098 (2014)

36. Simsek, Y: On q-deformed Stirling numbers. Int. J. Math. Comput. 15, 70-80 (2012)

37. Simsek, Y: Complete sum of products of $(h, q)$-extension of Euler polynomials and numbers. J. Differ. Equ. Appl. 16 1331-1348 (2010)

38. Simsek, Y: Identities associated with generalized Stirling type numbers and Eulerian type polynomials. Math. Comput. Appl. 18, 251-263 (2013)

39. Simsek, Y: Generating functions for generalized Stirling type numbers, array type polynomials, Eulerian type polynomials and their applications. Fixed Point Theory Appl. 2013, 87 (2013)

40. Simsek, Y: Functional equations from generating functions: a novel approach to deriving identities for the Bernstein basis functions. Fixed Point Theory Appl. 2013, 80 (2013)

41. Simsek, Y: Twisted $(h, q)$-Bernoulli numbers and polynomials related to twisted $(h, q)$-zeta function and $L$-function. J. Math. Anal. Appl. 324(2), 790-804 (2006)

42. Simsek, Y: Apostol type Daehee numbers and polynomials. Adv. Stud. Contemp. Math. 26(3), 555-566 (2016) 
43. Simsek, Y: Identities on the Changhee numbers and Apostol-Daehee polynomials. Adv. Stud. Contemp. Math. (2017, to appear)

44. Simsek, Y, Acikgoz, M: A new generating function of (q-) Bernstein-type polynomials and their interpolation function. Abstr. Appl. Anal. 2010, Article ID 769095 (2010)

45. Schikhof, WH: Ultrametric Calculus: An Introduction to p-Adic Analysis. Cambridge Studies in Advanced Mathematics, vol. 4. Cambridge University Press, Cambridge (1984)

46. Srivastava, HM: Some generalizations and basic (or q-) extensions of the Bernoulli, Euler and Genocchi polynomials. Appl. Math. Inf. Sci. 5, 390-444 (2011)

47. Srivastava, HM, Kim, T, Simsek, Y: $q$-Bernoulli numbers and polynomials associated with multiple $q$-zeta functions and basic L-series. Russ. J. Math. Phys. 12, 241-268 (2005)

48. https://en.wikipedia.org/wiki/Polynomial

49. Zwinderman, AH, Cleophas, TA, Cleophas, TJ, van der Wall, EE: Polynomial analysis of ambulatory blood pressure measurements. Neth. Heart J. 9(2), 68-74 (2001)

50. Vladimirov, VS, Volovich, IV, Zelenov, El: $p$-Adic Analysis and Mathematical Physics. Series on Soviet and East European Mathematics, vol. 1. World Scientific, Singapore (1994)

Submit your manuscript to a SpringerOpen ${ }^{\circ}$ journal and benefit from:

- Convenient online submission

Rigorous peer review

- Immediate publication on acceptance

- Open access: articles freely available online

- High visibility within the field

- Retaining the copyright to your article 